\title{
Lung volumes related to physical activity, physical fitness, aerobic capacity and body mass index in students
}

\author{
A. Mihailova and I. Kaminska \\ Daugavpils University, Daugavpils, Latvia
}

\begin{abstract}
The aim of this study was to estimate the correlation between the function parameters of the respiratory system and the amount of physical activity, body mass index and aerobic and physical fitness in Daugavpils University students. The study involved a group of 191 students of both sexes from Daugavpils University aged 20-36 years.

Students $(\mathrm{n}=191,123$ females and 68 males, mean age $24.02 \pm$ 0.24 years) underwent measurement of anthropometric values (height, weight), physical (PWC170 index) and aerobic fitness (predicted VO2max), spirometry (tidal volume, breathing frequency $(\mathrm{BF})$, minute ventilation (MV), expiratory reserve volume (ERV) and vital capacity (VC) and filled in International Physical Activity Questionnaire (IPAQ).

Both MV and VC were directly and statistically significantly associated with aerobic fitness, physical fitness and amount of physical activity. Vigorous PA independently was directly and statistically significantly associated with physical fitness and aerobic fitness. After adjusting for sex, BF was inversely and statistically significantly associated with physical activity amount in female. Both female and male had inverse and statistically significant association of $\mathrm{BMI}$ and relative $\mathrm{VO} 2$ max.

Reduced lung volumes were associated with lower aerobic fitness, lower physical fitness and lower amount of weekly physical activity. Healthier body mass index was associated with higher aerobic fitness (relative $\mathrm{VO} 2 \max )$ in both female and male.
\end{abstract}

\section{Introduction}

Pulmonary function is a long-term predictor for overall survival rates in both genders $[1,2]$. Schunemann et al. state the relation of pulmonary function with mortality in general population having results from prospective study with 29-year follow-up of the Buffalo Health Study cohort of people aged 20 to 89 years [1]. However, Sanatana et al. studied 97 men aged 67-78 years and study data showed a significant association between body composition, fat distribution, and lung function in elderly men [3]. Previous studies have investigated obesity being associated with altered lung function. It has been found that central obesity may be an important mediator of the relationship between altered lung function, adverse metabolic profiles and subsequent diabetes and CVD risk [4]. Rożek-Piechura K. et al. (2014) obtained that parameters of the respiratory system show significant correlations with body composition parameters (FFM, water) [5]. In obese individuals, structural changes of the thoracic-abdominal region lead to limited diaphragm mobility and rib movement, both essential for appropriate ventilation mechanics [4]. 
Table 1. Characteristics of research subjects.

\begin{tabular}{|c|c|c|}
\hline Parameter & Men $(\mathbf{n}=\mathbf{1 2 3})$ & Women $(\mathbf{n}=\mathbf{6 8})$ \\
\hline Age $($ years $)$ & $24.14 \pm 3.6$ & $23.81 \pm 2.7$ \\
\hline Height $(\mathrm{cm})$ & $167.15 \pm 6.2$ & $180.81 \pm 8.0$ \\
\hline Weight $(\mathrm{kg})$ & $77.4 \pm 10.5$ & $60.56 \pm 9.1$ \\
\hline BMI $\left(\mathrm{kg} / \mathrm{m}^{2}\right)$ & $21.6 \pm 2.7$ & $23.8 \pm 2.6$ \\
\hline
\end{tabular}

But many studies have not been adjusted to measuring physical activity levels or aerobic fitness, both of which are important determinants of respiratory function, too. Physical activity and fitness have important health promoting effects with respect to arteriosclerosis and coronary heart disease in particular [6]. Normal BMI and anthropometric indices do not serve as guarantee of physical fitness for university undergraduates who are involved in sedentary life style. Regular physical activity has a strong positive impact on physical fitness, particularly on aerobic capacity which is the most important health promoting component of physical fitness with respect to coronary heart disease [6]. Paulo R. et al. (2013) studied physical activity and respiratory function making corporal composition and spirometric values analysis. The sample consisted of 86 individuals, higher education students, with an average age of $21.3 \pm 2.4$ years, who were divided into two groups: the control group consisted of 28 sedentary subjects ( $20.9 \pm 1.3$ years), and the experimental group consisting of 58 subjects $(21.5 \pm 2.8$ years) who undertook supervised exercise [7]. According to Paulo R. et al. the students who performed supervised exercise had higher levels of body composition and lung function. Poor BMI and waist circumference values may lead to respiratory dysfunction in terms of ventilation and the respective lung volumes, limiting the practice of physical activity and increasing the probability of respiratory pathologies [7].

The studies used different body composition assessing methods, but nevertheless the results state association between body composition and lung function in different age groups. Therefore, in this study we sought to examine the relationship between lung function and different health-related parameters: body composition, physical activity (PA) and aerobic fitness in students of both sexes aged 20-36 years. In particular, we precisely quantified objectively measured body mass index (BMI), subjectively measured physical activity (PA) and aerobic fitness $\left(\mathrm{VO}_{2} \max \right)$ to describe the association between BMI, PA, aerobic fitness and lung function.

\section{Materials and methods}

\section{Participants}

The study involved a group of 191 students of both sexes (123 women and 68 men) from Daugavpils University (Daugavpils, Latvia) aged 20-36 years (mean age 24.02 \pm 0.24 years). All students were full-time students of Physiotherapy, Physical Education and Biology study programs. Subjects were included in investigation according to inclusion criteria: students of full-time programs in Physiotherapy, Physical Education and Biology, absence of contradictions to any of tests and absence of severe health problems). Mean $[ \pm \mathrm{SD}]$ weight of women was $60.56 \pm 9.1 \mathrm{~kg}$ and for men $-77.4 \pm 10.5 \mathrm{~kg}$. Other characteristics of subjects are listed in Table 1.

191 students underwent measurement of anthropometric values (height, weight), physical ( $\mathrm{PWC}_{170}$ index) and aerobic fitness (predicted $\mathrm{VO}_{2} \mathrm{max}$ ), spirometry (tidal volume, breathing frequency $(\mathrm{BF})$, minute ventilation (MV), expiratory reserve volume (ERV) and vital capacity (VC) measurements. 143 filled in the International Physical Activity Questionnaire (IPAQ). All participants were tested once attending the laboratory. Height was measured and was used 
for weight and BMI assessment that was done in the morning after light breakfast. This was followed by spirometry measurement in resting condition and $\mathrm{PWC}_{170}$ test on cycle ergometre and cardiopulmonary testing device.

\section{Methods}

Height was measured according to standardized procedure using body measuring tape ADE MZ10017. Height was measured to the nearest $1.0 \mathrm{~cm}$. Body weight and body mass index measures were obtained from the complex body composition assessment. Body composition was measured using bioelectrical impedance method using medically approved body composition monitor TANITA BC-420. Body weight and body mass index measures were taken to the nearest $0.1 \mathrm{~kg}$ and $0.1 \mathrm{~kg} / \mathrm{m}^{2}$ respectively.

Spirometry was assessed using cardiopulmonary testing system MasterScreenCPX (JAEGER) performing slow spirometry measurement. Actual and predicted values of tidal volume (VT), breathing frequency $(\mathrm{BF})$, minute ventilation $(\mathrm{MV})$, expiratory reserve volume (ERV) and vital capacity (VC) were measured to nearest 0.011 .

Cardiorespiratory fitness was assessed using cardiopulmonary testing system MasterScreenCPX (JAEGER) and cycle ergometer Ergoselect 100P (Ergoline, SN 2008000567) with breath-by-breath analysis of expired air. Subjects performed submaximal test based on the method of $\mathrm{PWC}_{170}$ testing with two power stages each of 5 minutes (Adams and Beam, 2008). Start power for both women and men was equivalent to $1 \mathrm{~W}$ per 1 kilogram of body weight for subject with normal BMI and $0.75 \mathrm{~W}$ per 1 kilogram of body weight for overweight subjects. Then power was increased according to the heart rate at the end of the first load stage. Maximal oxygen consumption was calculated in MasterScreenCPX program automatically. Additionally $\mathrm{PWC} 170$ index was calculated using standard equation: $\mathrm{PWC}_{170}=\mathrm{W}_{1}+\left(\mathrm{W}_{2}-\mathrm{W}_{1}\right) \times\left(170-\mathrm{f}_{1}\right) /\left(\mathrm{f}_{2}-\mathrm{f}_{1}\right)$, where $\mathrm{W}_{1}$ and $\mathrm{W}_{2}$ are the first and the second loads respectively and $f_{1}$ - heart rate at the end of the first load stage, $f_{2}$ - heart rate at the end of the second load stage. PWC170 index was taken as a basic variable for physical fitness assessment and maximal oxygen consumption was taken as basic variable for evaluation of aerobic capacity.

Physical activity was assessed with International Physical Activity Questionnaire (IPAQ) - Long Form. Three levels of physical activity were established according to IPAQ Scoring Protocol guidelines: low, moderate and high [8].

Statistical analysis was performed using IBM SPSS Statistics (version 20.0). Group differences were assessed with Chi-Square test. Differences between mean variables of independent samples were assessed using T-test and ANOVA, drawn at significant level of $0.05 \%$. Pearson correlation coefficient at significant level of $0.05 \%$ was estimated assessing correlations between variables.

\section{Results}

Mean BMI in women was $23.8 \pm 2.6 \mathrm{~kg} / \mathrm{m}^{2}$ and $21.6 \pm 2.7 \mathrm{~kg} / \mathrm{m}^{2}(p=0.0001)$. Most students had normal body weight and BMI in both genders: $80.49 \%$ in women and $73.53 \%$ in men. The difference between BMI groups in women and men was significant $(p=0.0001)$. More men fit the overweight (22.06\%) and obesity (4.41\%) group comparing with women who have in contrary $9.76 \%$ of subjects in underweight group and no one in obesity group.

Comparing actual VT values with predicted individual values (Table 2) it was assessed that both men and women had a prevalence of normal or higher VT in relation to predicted VT. But it's detected that $29.3 \%$ of women compared to only $7.4 \%$ in men had VT that was lower than their predicted value (Chi-Square $p<0.0001)$. That states that women had higher 
Table 2. Tidal volume evaluation according to predicted value in tested subjects $(n=191)$.

\begin{tabular}{|l|c|c|c|}
\hline Actual VT vs Predicted value & Men (\%) & Women (\%) & Total (\%) \\
\hline Decreased & 7.4 & 29.3 & 21.5 \\
\hline Normal or higher & 92.6 & 70.7 & 78.5 \\
\hline Total in column \% & 100.0 & 100.0 & 100.0 \\
\hline
\end{tabular}

Table 3. Minute ventilation evaluation according to predicted value in tested subjects $(\mathrm{n}=191)$.

\begin{tabular}{|l|c|c|c|}
\hline Actual MV vs Predicted value & Men (\%) & Women (\%) & Total (\%) \\
\hline Decreased & 41.8 & 60.7 & 53.9 \\
\hline Normal or higher & 58.2 & 39.3 & 46.1 \\
\hline Total in column \% & 100.0 & 100.0 & 100.0 \\
\hline
\end{tabular}

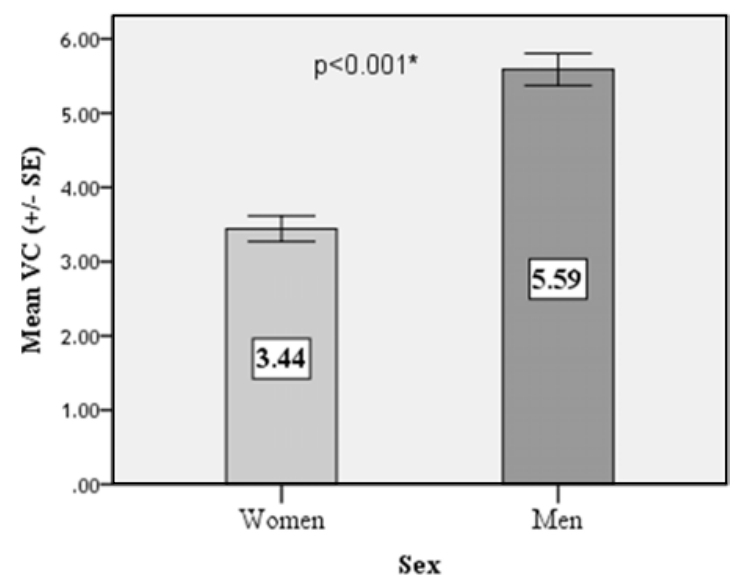

Figure 1. Mean vital capacity values in women $(n=123)$ and men $(n=68)$.

risk for lower VT than men. And analyzing the distribution of subject in groups according to evaluation of VT related to physiological norms $(350-750 \mathrm{ml})$ it was seen that mostly men (66.2\%) had tidal volume that was higher than the normal values and therefore $70.7 \%$ of women had normal VT, $11.4 \%$ had decreased VT values (VT $<350 \mathrm{ml}$ ).

Mean minute ventilation (MV) values in men were $12.47 \pm 3.2 l$ comparing with $8.05 \pm$ $4.6 l$ in women $(p<0.001)$. Evaluating MV according to predicted values it was observed that the distribution in groups differed statistically significantly $(p=0.013)$. Women had a prevalence of subjects with decreased MV that did not fit their individual predicted value, but $58.2 \%$ of men in contrary had normal or higher MV (Table 3).

Mean vital capacity values in men were $5.59 \pm 0.9 l$ comparing with $3.44 \pm 1.0 l$ in women $(p<0.001)$ (Fig. 1). Men had statistically significantly higher VC than women, but therefore mean value of $\mathrm{VC}$ in both genders fitted the normal values $(3-5 l)$. Stratifying by gender $79.4 \%$ of men comparing with $4.9 \%$ of women had increased $\mathrm{VC}$, but $30.1 \%$ of women had decreased VC. Women also had lower expiratory reserve volume (ERV) values comparing with men and $71.3 \%$ had decreased ERV comparing with individual predicted values. $78.1 \%$ of women had ERV lower than $1.5 \mathrm{l}$. In men ERV fitted predicted values in $59.1 \%$ of subjects and also was normal or higher in $70.2 \%$. 
Table 4. Correlation between lung volumes and $\mathrm{PWC}_{170}$ index and maximal oxygen consumption.

\begin{tabular}{|c|c|c|}
\hline Correlation coefficient $r$ & $\mathbf{P W C}_{\mathbf{1 7 0}}$ index & $\mathbf{V O}_{\mathbf{2}} \mathbf{m a x}$ \\
\hline VT & $r=0.485^{*}$ & $r=0.404^{*}$ \\
\hline ERV & $r=0.395^{*}$ & $r=0.368^{*}$ \\
\hline MV & $r=0.420^{*}$ & $r=0.367^{*}$ \\
\hline VC & $r=0.647^{* *}$ & $r=0.600^{* *}$ \\
\hline
\end{tabular}

$* \mathrm{p}<0.05, * * \mathrm{p}<0.01$

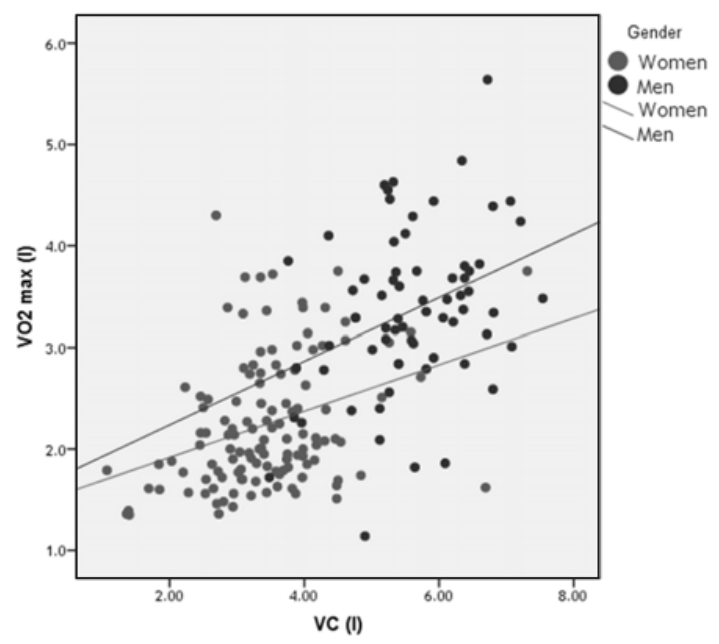

Figure 2. VC association with maximal oxygen consumption in both genders.

VT, ERV, MV and VC had statistically significant and positive correlation with physical fitness index $\mathrm{PWC}_{170}$ (Table 4). The same results lung volumes showed correlating statistically significantly and positively with maximal oxygen consumption $\left(\mathrm{VO}_{2}\right.$ max $)$ values (Table 4). Students with higher lung volumes (VT, ERV, MV, VC) had statistically significant higher physical and aerobic fitness.

$51.7 \%$ of students had moderate physical activity (PA) level, 34.3\% had low PA level and only $14.0 \%$ of students - high PA level. Women were less physically active than men $(p=0.001)$ having almost equal prevalence of low and moderate physical activity level (PAL) (44.0 and 45.0\%) comparing with $67.4 \%$ of men in moderate PAL. Both MV $(r=0.198, p<0.05$ and $r=0.194, p<0.05)$ and VC $(r=0.371, p<0.05$ and $r=0.307, p<0.05)$ were directly and statistically significantly associated with vigorous physical activity amount and total physical activity amount. Vigorous PA independently was directly and statistically significantly associated with physical fitness $(r=0.374, p<0.05)$ and aerobic capacity $(r=0.321, p<0.05)$. Walking and moderate PA amount did not show significant association to physical fitness and aerobic capacity.

After adjusting gender, correlation between $\mathrm{VC}$ and $\mathrm{VO}_{2}$ max was direct and statistically significant in both genders ( $r=0.331$ in women and $r=0.303$ in men) (Fig. 2). So those subjects of both genders who had higher $\mathrm{VC}$ had higher $\mathrm{VO}_{2} \max$ values that correspond to higher aerobic capacity. Both women $(r=-0.208, p<0.05)$ and men $(r=-0.337$, $p<0.05$ ) had inverse and statistically significant association of BMI and relative $\mathrm{VO}_{2}$ max (Fig. 3). This association shows that subjects of both genders with higher BMI had lower relative $\mathrm{VO}_{2}$ max and lower aerobic fitness. 


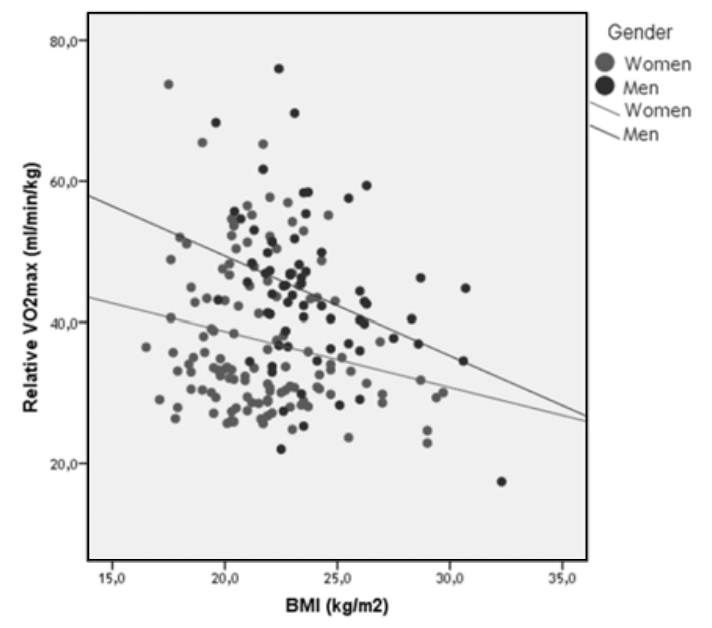

Figure 3. BMI association with relative maximal oxygen consumption in both genders.

\section{Discussion}

The purpose of this study was to estimate the correlation between the function parameters of the respiratory system and the amount of physical activity, body mass index and aerobic and physical fitness in Daugavpils University students. The result showed that greater lung volumes were associated with a higher level of physical fitness and aerobic capacity in students of Physiotherapy, Physical Education and Biology study programs. Higher vigorous physical activity amount and total physical activity amount were associated with higher minute ventilation and vital capacity. Weekly amount of vigorous physical activity was correlated positively with higher physical fitness and aerobic fitness. Taking into account that vigorous PA and lung volumes were associated both with physical fitness and aerobic capacity, we can state that physical fitness and aerobic capacity are the main factors of better lung function and this can be obtained maintaining high vigorous PA amount weekly.

Additionally, our finding showed that healthier body mass index was associated with higher aerobic capacity (relative $\mathrm{VO}_{2} \mathrm{max}$ ) in both women and men. As vital capacity was higher in subjects with higher $\mathrm{VO}_{2}$ max in both genders, we can conclude that in both women and men indirectly vital capacity is associated with BMI.

Our study findings are consistent with Maroos and Landor (2001) study finding. Maroos and Landor (2001) concluded that regular physical activity has a strong positive impact on physical fitness, particularly on aerobic capacity which is the most important health promoting component of physical fitness with respect to coronary heart disease. We have found not only positive association of PA and physical and aerobic capacity, but also association of PA and lung functions, specifically vital capacity.

With regard to body mass index evaluation Maroos and Landor (2001) investigating university undergraduates (University of Tartu) had the same results as we have. Most of the female and male students had normal BMI [6]. Our study group showed the same results and most students had normal body weight and BMI in both genders: $80.49 \%$ in women and $73.53 \%$ in men. In both Estonia and Latvia students have normal body mass index and this age group 20-36 years has healthy body composition.

As it was told before, we have also found an indirect association of BMI and vital capacity, according to their relation to aerobic capacity. Paulo et al. (2013) investigating the same study group - higher education students - have similar results, namely, there are some 
indicators that the higher the BMI, the worse the spirometry values, i.e. the more inadequate the body composition values and the more reduced are the lung volumes, reflecting a change of respiratory function, restricting daily function and eventually also physical activity practice [7].

\section{Conclusions}

Higher lung volumes are associated with higher aerobic fitness, higher physical fitness and higher amount of weekly physical activity in students. Healthier body mass index is associated with higher aerobic fitness (relative $\mathrm{VO}_{2} \mathrm{max}$ ) in students of both genders.

\section{References}

[1] H.J. Schunemann, J. Dorn, B.J.B. Grant, W. Winkelstein, M. Trevisan. Pulmonary function is a long-term predictor of mortality in the general population - 29-year followup of the Buffalo Health Study. CHEST, 118(3), 656 (2000).

[2] T.H. Beaty, C.A. Newill, B.H. Cohen, M.S. Tockman, S.H. Bryant, H.A. Spurgeon. Effects of pulmonary function on mortality. J Chronic Dis, 8, 703 (1985).

[3] H. Santana, E. Zoico, E. Turcato, P. Tosoni, L. Bissoli, M. Olivieri, O. Bosello, M. Zamboni. Relation between body composition, fat distribution, and lung function in elderly men. Am J Clin Nutr, 73(4), 827 (2001).

[4] L. Costa Melo, M.A. Mendonca da Silva, A.C. Nascimento Calles. Obesity and lung function: a systematic review. Einstein (São Paulo), 12(1) (2014).

[5] K. Rożek-Piechura. Z. Ignasiak, T. Sławińska, J. Piechura, T. Ignasiak. Respiratory function, physical activity and body composition in adult rural population. Ann Agric Environ Med, 21(2), 369 (2014).

[6] J. Maaroos, A. Landör. Anthropometric indices and physical fitness in university undergraduates with different physical activity. Anthropol Anz, 59(2), 157 (2001).

[7] R. Paulo, J. Petrica, J. Martins. Physical activity and respiratory function: corporal composition and spirometric values analysis. Acta Med Port, 26(3), 258-259 (2013).

[8] E. Patterson Guidelines for Data Processing and Analysis of the International Physical Activity Questionnaire (IPAQ). Retrieved May 29, 2015, from https://sites. google.com/site/theipaq/scoring-protocol (2010, November). 